\title{
Integer-valued definable functions
}

\author{
G. O. Jones, M. E. M. Thomas and A. J. Wilkie
}

\begin{abstract}
We present a dichotomy, in terms of growth at infinity, of analytic functions definable in the real exponential field which take integer values at natural number inputs. Using a result concerning the density of rational points on curves definable in this structure, we show that if a definable, analytic function $f:[0, \infty)^{k} \rightarrow \mathbb{R}$ is such that $f\left(\mathbb{N}^{k}\right) \subseteq \mathbb{Z}$, then either $\sup _{|\bar{x}| \leqslant r}|f(\bar{x})|$ grows faster than $\exp \left(r^{\delta}\right)$, for some $\delta>0$, or $f$ is a polynomial over $\mathbb{Q}$.
\end{abstract}

\section{Introduction}

The results presented in this note concern the growth at infinity of functions which are analytic and definable in the real field expanded by the exponential function and which take integer values on $\mathbb{N}$. They are descendants of a theorem of Pólya [8] from 1915 concerning integervalued, entire functions on $\mathbb{C}$. This classic theorem tells us that $2^{z}$ is, in some sense, the smallest non-polynomial function of this kind. More formally, let $m(f, r):=\sup \{|f(z)|:|z| \leqslant r\}$. Pólya's Theorem, refined in $[\mathbf{3}, \mathbf{9}]$, states the following.

THEOREM $1.1[9$, Theorem I]. If $f: \mathbb{C} \rightarrow \mathbb{C}$ is an entire function which satisfies both $f(\mathbb{N}) \subseteq$ $\mathbb{Z}$ and

$$
\limsup _{r \rightarrow \infty} \frac{m(f, r)}{2^{r}}<1
$$

then $f$ is a polynomial.

This result cannot be directly transferred to the real analytic setting (for example, consider the function $f(x)=\sin (\pi x)$ ). In fact, it appears that there are very few results of this character known for such functions (some rare examples may be found in [1]). We propose that a suitable setting in which to prove analogous results for real analytic functions is that of o-minimal expansions of the real field $\overline{\mathbb{R}}$, in which oscillating functions cannot be defined. We assume that the reader is familiar with the basic properties of such structures (see, for example, [2]). The following result holds in arbitrary o-minimal expansions of $\overline{\mathbb{R}}$ and is, to the authors' knowledge, the only result with the character of 1.1 already known for such structures.

TheOREM $1.2[\mathbf{1 0}$, Corollary 2.2]. Suppose that $f: \mathbb{R} \rightarrow \mathbb{R}$ is definable in an o-minimal expansion of $\overline{\mathbb{R}}$, that $k \in \mathbb{N}, c \in \mathbb{R}$ and that $|f(x)| \leqslant c x^{k}$, for large $x$. Suppose further that $f(\mathbb{N}) \subseteq \mathbb{Z}$. Then there exists a polynomial $\rho(x) \in \mathbb{Q}[x]$ such that $f(x)=\rho(x)$ for all sufficiently large $x$.

2010 Mathematics Subject Classification 03C64 (primary), $26 \mathrm{E} 05$ (secondary).

The first author was supported in part by The Manchester Centre for Interdisciplinary Computational and Dynamical Analysis (CICADA), EPSRC grant EP/E050441/1, and by an EPSRC Postdoctoral Fellowship, EPSRC grant EP/F043236/1. The second author was supported by the Zukunftskolleg, Universität Konstanz. 
We shall outline several results, in the direction of Theorem 1.1, for those functions which are definable in the structure $\mathbb{R}_{\exp }:=\langle\bar{R}$, exp $\rangle$. The main theorem we shall prove is as follows. For technical reasons (and, indeed, for a stronger result), we work in the expanded structure $\mathbb{R}_{\text {exp, } \sin \{[0,2 \pi]}:=\left\langle\overline{\mathbb{R}}, \exp , \sin _{[0,2 \pi]}\right\rangle$. Here, for a function $f$, we define the maximum function $M_{f}(r):=\sup \left\{|f(\bar{x})|: \bar{x} \in \overline{B_{r}(0)} \cap \operatorname{dom}(f)\right\}$, where $\overline{B_{r}(0)}$ denotes the closed ball of radius $r$ centred on the origin.

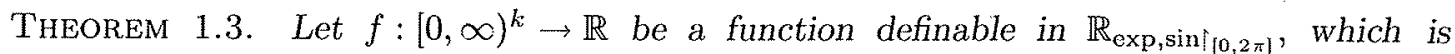
analytic and such that $f\left(\mathbb{N}^{k}\right) \subseteq \mathbb{Z}$. If, for all $\epsilon>0$, ultimately $M_{f}(t)<\exp \left(t^{*}\right)$, then $f$ is a polynomial over $\mathbb{Q}$.

It is worth noting that this is not an empty theorem. For example, for all $n \geqslant 2$,

$$
f_{n}(x)=\exp _{n}\left(2 \log _{n}(x)\right) \text { and } g_{n}(x)=\exp _{n+1}\left(\frac{1}{2} \log _{n}(x)\right)
$$

are analytic functions definable in $\mathbb{R}_{\exp }$ which ultimately grow slower than $\exp \left(x^{\epsilon}\right)$, for every $\epsilon>0$, but faster than all polynomials. This theorem tells us that such functions cannot take integer values at every natural number. Had we defined $f_{n}$ and $g_{n}$ with exponentials and logarithms to base 2 instead of $e$, then the same conclusion would also apply. In this instance, it is clear to see that there would still be infinitely many natural numbers at which these functions would take integer values.

As we shall see later, a more general version of Theorem 1.3 also holds. If $\mathbb{N}^{k}$, for $k \in \mathbb{N}$, is replaced by any subset of $\mathbb{R}^{k}$ of the form $\prod_{i=1}^{k}\left(s_{i} \mathbb{N}+u_{i}\right) \cap[0, \infty)^{k}$, where $s_{i}$ and $u_{i}$ lie in a fixed subfield $K$ of $\mathbb{R}$ and $s_{i}>0$, for all $i \in\{1, \ldots, k\}$, we may conclude that $f$ is a polynomial over $K$.

In order to prove the main theorem, we shall make use of a result concerning the density of rational points on sets definable in the structure $\mathbb{R}_{\exp , \sin [[0,2 * x]}$, outlined in Section 2. A number of requisite lemmas, leading up to the proof of Theorem 1.3, follow in Section 3.

\section{The density of rational points}

In this section, we state the principal result which we shall need in order to prove our main theorem. It falls into a general program of bounding the density of rational and algebraic points on subsets of $\mathbb{R}^{n}$, with a view to applications to transcendental number theory, following the work of Pila $[5,6]$ and Pila and Wilkie [7]. The particular result which we shall use here concerns the density of rational points on sets definable in $\mathbb{R}_{\text {exp, } \sin [0,2 \pi]}$. This is a specific instance of a result which also applies to points from any number field over $\mathbb{R}$ and to sets definable in any model complete expansion of $\overrightarrow{\mathbb{R}}$ by a Pfaffian chain, but we shall not need this level of generality here (for more details, see [4]).

In order to state this result, we first need a few definitions.

Definition 1. For any $a / b \in \mathbb{Q}$ in its simplest form, that is, with $\operatorname{gcd}(a, b)=1$, and with $b>0$, we define its height $H(a / b)$ to be $\max \{|a|, b\}$. The height of a finite tuple of rationals $\left(q_{1}, \ldots, q_{m}\right)$ is defined to be $\max _{1 \leqslant i \leqslant m}\left\{H\left(q_{i}\right)\right\}$. For any set $X \subseteq \mathbb{R}^{n}$, let $X(\mathbb{Q})$ denote its subset of points with rational coordinates and let $X(\mathbb{Q}, T)$ denote $\{\bar{q} \in X(\mathbb{Q}) \mid H(\bar{q}) \leqslant T\}$. We can define a counting function thus:

$$
N(X, T):=|X(\mathbb{Q}, T)|
$$

The theorem that we shall use may then be stated as follows. 
Theorem 2.1 [4, Corollary 4.5]. Suppose that $I \subseteq \mathbb{R}$ is an interval and that $f: I \rightarrow \mathbb{R}$ is a transcendental function definable in $\mathbb{R}_{\exp , \sin \{[0,2 \pi]}$. Let $X$ be the graph of $f$. There exist $c(X), \gamma(X)>0$ such that, for all $T \geqslant e$,

$$
N(X, T) \leqslant c(\log T)^{\gamma}
$$

\section{Integer-valued functions}

The result here which makes use of Theorem 2.1 is the following proposition for integer-valued functions of one variable.

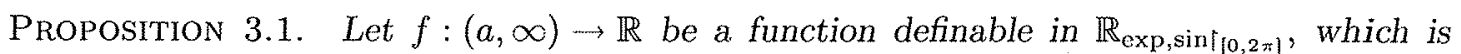
analytic and such that $f(n) \in \mathbb{Z}$, for all $n \in \mathbb{N} \cap(a, \infty)$. If, for all $\epsilon>0$, ultimately $f(t)<$ $\exp \left(t^{\epsilon}\right)$, then $f$ is a polynomial over $\mathbb{Q}$.

Proof. By analyticity of $f$ and Theorem 1.2 , in order to show that $f \in \mathbb{Q}[X]$, it is enough to show that $f$ is algebraic.

Therefore, let us suppose, for a contradiction, that $f$ is transcendental. In this case, we may apply Theorem 2.1 to see that, for $X$ the graph of $f$, there are $c, \gamma>0$ such that

$$
N(X, T) \leqslant c(\log T)^{\gamma},
$$

for all sufficiently large $T \in \mathbb{N}$.

Now let $\epsilon<1 / \gamma$. The growth condition on $f$ gives us that $f(t)<\exp \left(t^{\epsilon}\right)$, for all $t$ greater than some natural number $N_{\epsilon}>a$.

We may now choose $T \in \mathbb{N}$ large enough that $\log T \leqslant T^{\epsilon}$, and both the above inequality and

$$
(\log T)^{1 / c}>c(\log T)^{\gamma}+1+N_{\epsilon}
$$

hold. For such a $T$, we have

$$
\left|\mathbb{N} \cap\left(N_{\epsilon},(\log T)^{1 / \epsilon}\right]\right|>c(\log T)^{\gamma} .
$$

By the conditions imposed, all $n \in \mathbb{N} \cap\left(N_{\epsilon},(\log T)^{1 / \epsilon}\right]$ have $H((n, f(n))) \leqslant T$ and consequently $N(X, T)>c(\log T)^{\gamma}$, but this is a contradiction to the first inequality. Therefore, $f$ must be algebraic and hence a polynomial over $\mathbb{Q}$.

REMARK. Let us suppose that $f:(a, \infty) \rightarrow \mathbb{R}$ is an analytic, non-polynomial function definable in $\mathbb{R}_{\exp , \sin \left\lceil_{[0,2 \pi]}\right.}$. Moreover, suppose that, for all $\epsilon>0$, we are given $N_{c}>0$ such that $f(t)<\exp \left(t^{\epsilon}\right)$ on $\left(N_{\epsilon}, \infty\right)$. Proposition 3.1 tells us that $f$ cannot be integer-valued, that is, for some $n \in \mathbb{N} \cap(a, \infty), f(n) \notin \mathbb{Z}$. However, we can be somewhat more precise than this. The exponent $\gamma$ given to us by Theorem 2.1 may be calculated explicitly in terms of the complexity of the function $f$. Consequently, the proof of Proposition 3.1 shows that it is possible to find $N \in\left[0, \infty\right.$ ), in terms of $f$ and $N_{\epsilon}$ (for some fixed $\epsilon<1 / \gamma$ ), such that there is some $n \in \mathbb{N}$ with $n<N$ and $f(n) \notin \mathbb{Z}$.

The following is an easy, technical corollary which we shall need later.

Corollary 3.2. Let $K$ be a fixed subfield of $\mathbb{R}$. Let $f:(a, \infty) \rightarrow \mathbb{R}$ be an analytic function definable in $\mathbb{R}_{\exp , \sin _{[0,2 \pi}}$ which has the property that $f(s n+u) \in \mathbb{Z}$, for some $s, u \in K$ with $s>0$, and all $n \in \mathbb{N}$ such that $s n+u \in(a, \infty)$. If, for all $\epsilon>0$, ultimately $f(t)<\exp \left(t^{c}\right)$, then $f$ is a polynomial over $K$. 
Proof. Set $q(t):=s t+u$, for $t \in \mathbb{R}$. It is easy to see that the function $f \circ q:((a-u) / s$, $\infty) \rightarrow \mathbb{R}$ satisfies the hypotheses of Proposition 3.1. Therefore, $f \circ q$ is a polynomial over $\mathbb{Q}$ and hence $f=f \circ q \circ q^{-1}$ is a polynomial over $K$.

Now we wish to consider higher dimensions. Recall that, for a function $f$, we define $M_{f}(r):=\sup \left\{|f(\bar{x})|: \bar{x} \in \overline{B_{r}(0)} \cap \operatorname{dom}(f)\right\}$. We shall also need the following well-known and important fact. Any polynomial $p \in \mathbb{R}\left[X_{1}, \ldots, X_{k}\right]$, which has the property that $p\left(\mathbb{Z}^{k}\right) \subseteq \mathbb{Z}$, must be a $\mathbb{Z}$-linear combination of products of binomial coefficients $\left(\begin{array}{c}X_{i} \\ l\end{array}\right)$, for $i \in\{1, \ldots, k\}$, $l \in\{0, \ldots, \operatorname{deg}(p)\}$. Consequently, it is in fact a polynomial over $\mathbb{Q}$.

First, we shall prove a general lemma concerning definable analytic functions in several variables with polynomial growth, a natural extension to Theorem 1.2.

Lemma 3.3. Let $f:[0, \infty)^{k} \rightarrow \mathbb{R}$ be a function definable in an o-minimal expansion $\tilde{\mathbb{R}}$ of $\overline{\mathbb{R}}$, which is analytic and such that $f\left(\mathbb{N}^{k}\right) \subseteq \mathbb{Z}$. If there is a polynomial $p \in \mathbb{R}[X]$ such that ultimately $M_{f}(t)<p(t)$, then $f$ is a polynomial over $\mathbb{Q}$.

Proof. We proceed by induction on $k$. The base case is given to us by Theorem 1.2 .

Now suppose that the statement of the lemma holds for $k-1$ in place of $k$, and let $f:[0, \infty)^{k} \rightarrow \mathbb{R}$ satisfy the hypotheses of the lemma.

For each fixed $x_{1} \in[0, \infty)$, define the function $f_{x_{1}}:[0, \infty)^{k-1} \rightarrow \mathbb{R}$ by

$$
f_{x_{1}}\left(x_{2}, \ldots, x_{k}\right)=f\left(x_{1}, x_{2}, \ldots, x_{k}\right) .
$$

These functions are definable in $\tilde{\mathbb{R}}$ and are analytic. Moreover, if $x_{1} \in \mathbb{N}$, then both $f_{x_{1}}\left(\mathbb{N}^{k-1}\right) \subseteq$ $\mathbb{Z}$ and $M_{f_{x_{1}}}(t)<M_{f}(t)<p(t)$, for $t$ sufficiently large (depending on $x_{1}$ ). By the inductive hypothesis, it follows that $f_{n} \in \mathbb{Q}\left[X_{2}, \ldots, X_{k}\right]$, for all $n \in \mathbb{N}$. Let $d_{n}:=\operatorname{deg}\left(f_{n}\right)$, for all $n \in \mathbb{N}$, and let $d:=\operatorname{deg}(p)$. Since $M_{f_{n}}(t)<p(t)$, for all $t$ sufficiently large, we must have that the degrees $d_{n}$ are bounded by $d$, for all $n \in \mathbb{N}$.

We now define the following subset of $[0, \infty)$.

$$
Z:=\left\{x_{1} \in[0, \infty) \mid f_{x_{1}} \in \mathbb{R}\left[X_{2}, \ldots, X_{k}\right] \text { with } \operatorname{deg} f_{x_{1}} \leqslant d\right\} .
$$

Since $\mathbb{N} \subseteq Z$ and $Z$ is definable, we must have that $Z$ contains some unbounded interval $(b, \infty)$, by o-minimality. The function $f$ is then a polynomial in $x_{2}, \ldots, x_{k}$ on $(b, \infty) \times[0, \infty)^{k-1}$, of degree at most $d$. Its coefficients $a_{\alpha}$ depend definably on $x_{1}$, for $|\alpha| \leqslant d$. Moreover, as $f_{n}$ is an integer-valued polynomial over $\mathbb{Q}$ of degree $\leqslant d$, for each $n \in \mathbb{N}$, the definable function $d ! a_{\alpha}$ takes integer values at every natural number $x_{1}>b$, for all $|\alpha| \leqslant d$.

Let us enumerate the indices $\alpha$ with $|\alpha| \leqslant d$ as $\alpha_{0}, \ldots, \alpha_{D-1}$, where $D:=\left(\begin{array}{c}d+k-1 \\ d\end{array}\right)$. For each $i \in\{0, \ldots, D-1\}$, we can write $\alpha_{i}$ as $\left(\alpha_{i, 2}, \ldots, \alpha_{i, k}\right)$. Further, let $p_{l}$ denote the $l$ th prime number. With this notation, we have the following equation:

$$
M\left(\begin{array}{c}
a_{\alpha_{0}}\left(x_{1}\right) \\
\vdots \\
a_{\alpha_{D-1}}\left(x_{1}\right)
\end{array}\right)=\left(\begin{array}{c}
f\left(x_{1}, 1, \ldots, 1\right) \\
\vdots \\
f\left(x_{1}, p_{2}^{D-1}, \ldots, p_{k}^{D-1}\right)
\end{array}\right),
$$

where $M$ is the Vandermonde matrix with $(i, j)$ th entry

$$
\left(p_{2}^{\alpha_{j, 2}} \cdots p_{k}^{\alpha_{j, k}}\right)^{i}
$$

for $i, j \in\{0, \ldots, D-1\}$. This matrix has non-zero determinant and therefore we can find a polynomial expression for each of the coefficients $a_{\alpha_{i}}, i \in\{0, \ldots, D-1\}$, in terms of 
$f\left(x_{1}, p_{2}^{j}, \ldots, p_{k}^{j}\right)$, for $j \in\{0, \ldots, D-1\}$. Since, for all $j \in\{0, \ldots, D-1\}$,

$$
\begin{aligned}
\left|f\left(x_{1}, p_{2}^{j}, \ldots, p_{k}^{j}\right)\right| & \leqslant M_{f}\left(\left(x_{1}^{2}+p_{2}^{2 j}+\ldots+p_{k}^{2 j}\right)^{1 / 2}\right) \\
& \leqslant p\left(\left(x_{1}^{2}+p_{2}^{2 j}+\ldots+p_{k}^{2 j}\right)^{1 / 2}\right),
\end{aligned}
$$

which is bounded by a polynomial in $x_{1}$, we must therefore have that each coefficient $a_{\alpha_{i}}$, with $i \in\{0, \ldots, D-1\}$, is also polynomially bounded.

The coefficients therefore satisfy the hypotheses of Proposition 3.1 and hence are polynomials over $\mathbb{Q}$ in $x_{1}$ on $(b, \infty)$. Consequently, $f$ is a polynomial over $\mathbb{Q}$ in $(b, \infty) \times[0, \infty)^{k-1}$. Finally, we may use the analyticity of $f$ to conclude that $f \in \mathbb{Q}[\bar{X}]$, as it agrees with a polynomial on an open subset of $[0, \infty)^{k}$.

Now we come to the proof of the main theorem. In the course of the following, we shall use the following standard notation. For two functions $f, g:(0, \infty) \rightarrow \mathbb{R}$, we write $f \sim g$ to mean that $\lim _{t \rightarrow \infty}(f(t) / g(t))=1$.

Proof of Theorem 1.3. We begin by making a change of variables and consider $f \uparrow_{(0, \infty)^{k}}$ as a function in the polar coordinates $(r, \bar{\theta})$, where $r \in(0, \infty)$ and $\bar{\theta}=\left(\theta_{1}, \ldots, \theta_{k-1}\right) \in[0, \pi / 2]^{k-1}$. Note that this is still a function definable in $\mathbb{R}_{\text {exp, } \sin [[0,2 \pi]}$ and that the change of coordinates is analytic.

For every $\bar{\theta} \in[0, \pi / 2]^{k-1}$, we define a corresponding one-variable function $f_{\bar{\theta}}:(0, \infty) \rightarrow \mathbb{R}$ by $f_{\bar{\theta}}(r)=f(r, \bar{\theta})$, which is analytic and definable in $\mathbb{R}_{\exp , \sin \left[{ }_{[0,2 \pi]}\right.}$. In addition, for fixed $\bar{\theta}$, the hypotheses on $f$ give that, for any $\epsilon>0,\left|f_{\bar{\theta}}(t)\right|<\exp \left(t^{\epsilon}\right)$, for sufficiently large $t$, independent of $\bar{\theta}$.

Now let us define $\Theta$ to be the set of those directions $\bar{\theta}$ for which the line passing through the origin with direction $\bar{\theta}$ also passes through a point of $\mathbb{N}^{k} \backslash\{\overline{0}\}$ (and hence through infinitely many points in this set). This set $\Theta$ is dense in $[0, \pi / 2]^{k-1}$. For every $\bar{\theta} \in \Theta$, the corresponding function $f_{\bar{\theta}}$ has the property that, for some $s=s(\bar{\theta}) \in \mathbb{R} \cap \overline{\mathbb{Q}}$, where $\overline{\mathbb{Q}}$ is the field of algebraic numbers over $\mathbb{Q}, f_{\bar{\theta}}(s n) \in \mathbb{Z}$, for any $n \in \mathbb{N}$. Consequently, by applying Corollary 3.2 , we see that, for every $\bar{\theta} \in \Theta$, the function $f_{\bar{\theta}}$ is a polynomial in $r$ over $\mathbb{R} \cap \overline{\mathbb{Q}}$ of some degree $d_{\bar{\theta}}$.

Let us define a function $\lambda:[0, \pi / 2]^{k-1} \rightarrow \mathbb{R} \cup\{-1\}$ by the following:

$$
\bar{\theta} \mapsto \begin{cases}\lambda & \text { such that there exists } c \in \mathbb{R} \text { with } f_{\bar{\theta}}(r) \sim c r^{\lambda} ; \\ -1 & \text { otherwise. }\end{cases}
$$

In $\mathbb{R}_{\exp ,\left.\sin \right|_{[0,2 \pi]}}$, this map is definable and therefore there is a cell decomposition $\mathcal{C}$ of $[0, \pi / 2]^{k-1}$ on every cell of which this map is continuous. Moreover, for all $\bar{\theta} \in \Theta, \lambda(\bar{\theta})=d_{\bar{\theta}} \in \mathbb{N}$. Since $\Theta$ is dense in $[0, \pi / 2]^{k-1}$, the exponent function $\lambda$ must therefore be constant and non-negative on each open cell of $\mathcal{C}$. Consequently, there is a bound $d \in \mathbb{N}$ on the values $\lambda$ takes on all of $[0, \pi / 2]^{k-1}$, except possibly on a set of lower dimension.

Now let us define the following set of directions:

$$
\Theta^{\prime}:=\left\{\bar{\theta} \in\left[0, \frac{\pi}{2}\right]^{k-1} \mid f_{\bar{\theta}} \text { is a polynomial of degree at most } d\right\} .
$$

This set is definable and contains all of $\Theta$, except possibly a set of lower dimension, and is therefore also dense in $[0, \pi / 2]^{k-1}$. By definable choice, we may choose definable coefficient functions $c_{0}(\bar{\theta}), \ldots, c_{d}(\bar{\theta})$ for all $\bar{\theta} \in \Theta^{\prime}$. Moreover, by taking an analytic cell decomposition of $[0, \pi / 2]^{k-1}$, we may assume that these coefficient functions are in fact analytic on $\Theta^{\prime} \backslash D^{\prime}$, where $D^{\prime}$ is some set of lower dimension; that is, they are analytic on a dense, definable set. 
For each $\bar{\theta} \in \Theta^{\prime} \backslash D^{\prime}, f_{\bar{\theta}}^{(d)}(r)=d ! c_{d}(\bar{\theta})$. However, $f_{\bar{\theta}}^{(d)}(r)$ is just $\left(\partial^{d} f / \partial r^{d}\right)(r, \bar{\theta})$, which is analytic on $(0, \infty) \times[0, \pi / 2]^{k-1}$. Therefore, for any $\bar{\phi} \in[0, \pi / 2]^{k-1}$,

$$
\lim _{\bar{\theta} \rightarrow \bar{\phi}} c_{d}(\bar{\theta})=\frac{1}{d !} \frac{\partial^{d} f}{\partial r^{d}}(\bar{\phi})
$$

and hence $c_{d}$ has an analytic continuation to each $\bar{\phi} \in D^{\prime}$ and is bounded on all of $[0, \pi / 2]^{k-1}$.

Now, for all $\bar{\theta} \in \Theta^{\prime} \backslash D^{\prime}, f_{\bar{\theta}}^{(d-1)}(r)=d ! c_{d}(\bar{\theta}) r+(d-1) ! c_{d-1}(\bar{\theta})$. As before, the left-hand side is analytic and, as we now have that $c_{d}$ is bounded on $[0, \pi / 2]^{k-1}$, we may also conclude similarly that $c_{d-1}$ has an analytic continuation to each $\bar{\phi} \in D^{\prime}$ and is bounded on all of $[0, \pi / 2]^{k-1}$. Continuing in this vein, we have that all the coefficient functions $c_{i}$ have an analytic continuation to each $\bar{\phi} \in D^{\prime}$, and hence are bounded on $[0, \pi / 2]^{k-1}$, each by some $C_{i}>0$, say.

Consequently, the function $c_{0}(\bar{\theta})+\ldots+c_{d}(\bar{\theta}) r^{d}$ is analytic on $(0, \infty) \times[0, \pi / 2]^{k-1}$ (where we are now considering the analytic continuations of the functions $c_{i}$ ). As it agrees with $f$ on the dense open set $(0, \infty) \times \Theta^{\prime} \backslash D^{\prime}$, we therefore have this representation of $f$ on all of $(0, \infty) \times[0, \pi / 2]^{k-1}$. Observe then that, for all $r \in(0, \infty)$, we have $M_{f}(r) \leqslant C_{0}+\ldots+C_{d} r^{d}$. Consequently, $f$ satisfies the hypotheses of Lemma 3.3 and hence $f$ is a polynomial over $\mathbb{Q}$.

Finally, in an entirely analogous manner to the proof of Corollary 3.2, we may also conclude the following.

Corollary 3.4. Let $K$ be a fixed subfield of $\mathbb{R}$. Let $f:[0, \infty)^{k} \rightarrow \mathbb{R}$ be a function definable

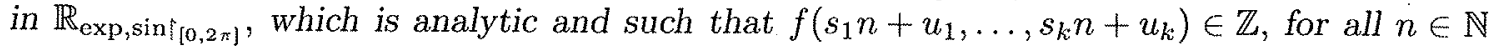
such that $s_{i} n+u_{i} \geqslant 0$, where $s_{i}, u_{i} \in K$ with $s_{i}>0$, for all $i \in\{1, \ldots, k\}$. If, for all $\epsilon>0$, ultimately $M_{f}(t)<\exp \left(t^{\epsilon}\right)$, then $f$ is a polynomial over $K$.

We close with a remark concerning the proof of Theorem 1.3. In order to demonstrate that a function $f:[0, \infty)^{k} \rightarrow \mathbb{R}$ satisfying the hypotheses of the theorem grows polynomially in $r$, we considered a dense set of lines through the origin, each passing through infinitely many points of $\mathbb{N}^{k}$. It would be of interest to know whether an analogous approach to our proof of Lemma 3.3, considering the restriction of $f$ only to those hyperplanes defined by fixing one coordinate to be an element of $\mathbb{N}$, would be sufficient. We believe that even the following question, for functions of two variables, is still open.

QUESTION 1. Let $f:[0, \infty)^{2} \rightarrow \mathbb{R}$ be an analytic function definable in an o-minimal expansion of $\overline{\mathbb{R}}$, with the property that, for each $n \in \mathbb{N}, f(n, \cdot)$ is a polynomial and $f(\cdot, n)$ is a polynomial. Is $f$ necessarily a polynomial?

\section{References}

1. D. H. Armitage, 'On harmonic functions which take integer values on integer lattices', Math. Proc. Cambridge Philos. Soc. 85 (1979) 69-77.

2. L. VAN DEN DRIES, Tame topology and o-minimal structures, London Mathematical Society Lecture Note Series 248 (Cambridge University Press, 1998).

3. G. H. Hardy, 'On a theorem of Mr. G. Pólya', Proc. Cambridge Philos. Soc. 19 (1917) 60-63.

4. G. O. JONES and M. E. M. ThOMAS, "The density of algebraic points on certain Pfafian surfaces", Q. J. Math. (2011) doi:10.1093/qmath/har011.

5. J. PILA, 'Geometric postulation of a smooth function and the number of rational points', Duke Math. J. 63 (1991) 449-463.

6. J. PILA, 'Integer points on the dilation of a subanalytic surface', Q. J. Math. 55 (2004) 207-223.

7. J. PILA and A. J. WILKIE, 'The rational points of a definable set', Duke Math. J. 133 (2006) 591-616.

8. G. PólYA, 'Ueber ganzwertige ganze Funktionen', Rend. Circ. Mat. Palermo 40 (1915) 1-16.

9. G. PólYA, 'Über ganze ganzwertige Funktionen', Nachr. Ges. Wiss. Göttingen (1920) 1-10.

10. A. J. WiLKIE, 'Diophantine properties of sets definable in o-minimal structures', J. Symbolic Logic 69 (2004) $851-861$. 
G. O. Jones

Centre for Interdisciplinary Computational and Dynamical Analysis (CICADA)

University of Manchester

Oxford Road

Manchester

M13 9PL

United Kingdom

gareth.jones-3@manchester.ac.uk
M. E. M. Thomas

Zukunftskolleg

Fachbereich Mathematik und Statistik

Fach 216

Universität Konstanz

D-78457 Konstanz

Germany

margaret.thomas@wolfson.oxon.org

\section{A. J. Wilkie}

School of Mathematics

University of Manchester

Oxford Road

Manchester

M13 9PL

United Kingdom

awilkie@maths.manchester.ac.uk 